\title{
Durable Response to PD1 Inhibitor Pembrolizumab in a Metastatic, Metaplastic Breast Cancer
}

\author{
Elan Gorshein ${ }^{a} \quad K^{2}$ Matsuda ${ }^{b} \quad$ Gregory Riedlinger $^{b} \quad$ Levi Sokol $^{c}$ \\ Lorna Rodriguez-Rodriguez $^{\mathrm{d}} \quad$ Firas Eladoumikdachi $^{\mathrm{e}}$ Miral Grandhi ${ }^{\mathrm{e}}$ \\ Shridar Ganesan ${ }^{a}$ Deborah L. Toppmeyer ${ }^{a} \quad$ Lindsay Potdevin $^{\mathrm{e}}$ \\ Kathleen Toomey ${ }^{f}$ Kim M. Hirshfielda Nancy Chan ${ }^{a}$ \\ aDivision of Medical Oncology, Department of Medicine, Rutgers Robert Wood Johnson \\ Medical School, Rutgers Cancer Institute of New Jersey, New Brunswick, NJ, USA; \\ bepartment of Pathology and Laboratory Medicine, Rutgers Robert Wood Johnson \\ Medical School, Rutgers Cancer Institute of New Jersey, New Brunswick, NJ, USA; \\ 'Department of Radiology, Rutgers Robert Wood Johnson Medical School, New Brunswick, \\ NJ, USA; dDepartment of Gynecologic Oncology, Rutgers Robert Wood Johnson Medical \\ School, Rutgers Cancer Institute of New Jersey, New Brunswick, NJ, USA; eDivision of \\ Surgical Oncology, Department of Surgery, Rutgers Robert Wood Johnson Medical School, \\ Rutgers Cancer Institute of New Jersey, New Brunswick, NJ, USA; fDivision of Medical \\ Oncology, Department of Medicine, Rutgers Robert Wood Johnson Medical School, \\ Steeplechase Cancer Center, Somerville, NJ, USA
}

\section{Keywords}

Metaplastic breast cancer - Exceptional response · Immunotherapy · Program death ligand-1

\begin{abstract}
Metaplastic breast cancer (MBC) is a rare and aggressive subtype of breast cancer. Tumor characteristics typically feature estrogen receptor, progesterone receptor, and HER2-negative, triple-negative breast cancer (TNBC), with a poorer prognosis relative to pure invasive ductal or lobular disease. Resistance to chemotherapy often leads to local recurrence and distant metastasis. Genomic profiling has identified multiple molecular abnormalities that may translate to targetable therapies in MBC. These tumors are known to display higher PDL1 expressivity than other subtypes of breast cancer, and disease control with pembrolizumab and chemotherapy has been documented. We identify a patient with metastatic, metaplastic
\end{abstract}

Elan Gorshein, Lorna Rodriguez-Rodriguez, and Kim M. Hirshfield: Contribution during his/her time at Rutgers Cancer Institute of New Jersey. 
TNBC, with mesenchymal components and osseous differentiation, who completed 2 years of pembrolizumab treatment and has remained without evidence of disease after 32 months of observation, while maintaining good quality of life. Future efforts should focus on immunotherapy response with respect to the various subtypes of $M B C$, and treatment should continue to be incorporated in clinical trials to maximize disease response.

(C) 2021 The Author(s).

Published by S. Karger AG, Basel

\section{Introduction}

Breast cancer is the most common female malignancy and represents the second leading cause of cancer-related death in women. The disease includes a heterogeneous group of tumors with 4 distinct subtypes characterized on the basis of molecular features for estrogen or progesterone receptor, human epidermal growth factor 2 (HER2), or triple-negative breast cancer (TNBC), which lacks overexpression of estrogen or progesterone receptor and HER2. TNBC comprises $10-20 \%$ of breast cancer subtypes but has higher rates of local and systemic disease relapse, as well as greater breast cancer-related mortality. Furthermore, the pathologic characterization of breast cancer is diverse and varies in microscopic appearance, prognosis, and response to therapy.

Metaplastic carcinoma of the breast (MBC) is a rare subtype of invasive breast cancer that is associated with distinct histopathologic and molecular phenotypes [1]. It often presents as high-grade carcinoma in the setting of a heterogeneous large tumor that is triple negative and aggressive. MBC is typically chemoresistant in nature, with a high risk of disease recurrence, and poor overall survival relative to pure invasive ductal or lobular disease [2]. MBC tends to metastasize hematogenously, with lung and bone involvement more common than lymph node invasion [2]. In the metastatic setting, medial survival is approximately 8 months [2]. Given the rarity of this disease, research to improve treatment regimens has been limited, and standard treatment options for more common types of disease are frequently implemented, despite little data demonstrating improved outcomes with systemic neoadjuvant or adjuvant chemotherapy [2]. Targeted therapy for molecular alterations is an area of active investigation $[3,4]$.

We identify a patient with metastatic, MBC with a durable response to pembrolizumab therapy. Future efforts should aim to incorporate immunotherapy in clinical trials to maximize disease response.

\section{Case Presentation}

A 72-year-old postmenopausal, multiparous female was referred for Precision Medicine consultation after being diagnosed with stage IV MBC. Her past medical history was significant for chronic colitis with daily loose stools, former tobacco use, and emphysema, but no family history of malignancy. She had no prior mammograms and presented with a rapidly enlarging mass of her right breast.

On histology, the carcinoma exhibited biphasic pattern, comprised by an epithelial component and a mesenchymal component, along with osseous/bone formation (shown in Fig. 1a). On higher power view, the carcinoma cells from the epithelial component were poorly differentiated, with high-grade features characterized by poor tubule formation, and pleomorphic nuclei with many mitotic figures. The cells in the mesenchymal component exhibited spindle cell morphology (shown in Fig. 1b). These features were characteristic of MBC. In the area with bone formation (shown in Fig. 1c), the poorly differentiated carcinoma

\section{Karger' ${ }^{\prime \prime}$}




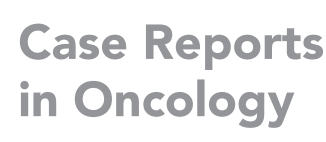

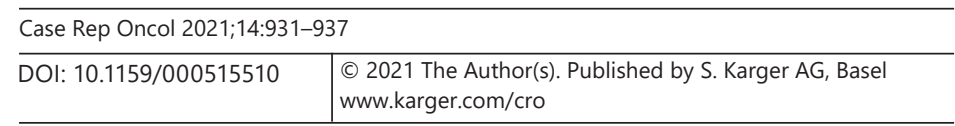

Gorshein et al.: PD1 Inhibitor for Metaplastic Breast Cancer
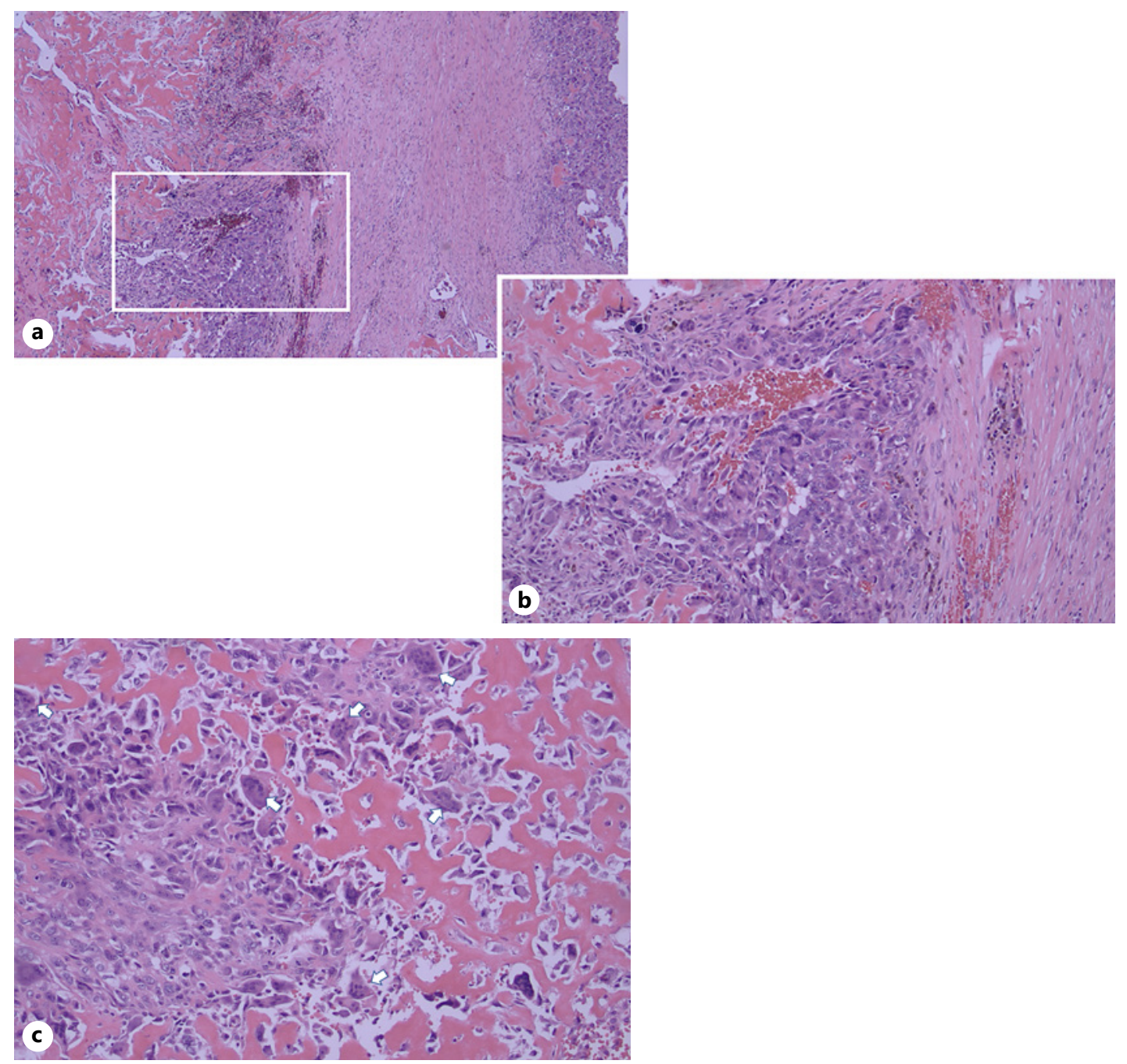

Fig. 1. Histopathologic findings. Low power view at $\times 40$ magnification (a), and magnified view of the rectangle area at $\times 200$ magnification (b). a Epithelial component and mesenchymal component along with area of bone formation are demonstrated. The carcinoma cells exhibit high-grade features: poor tubule formation, pleomorphic nuclei, and many mitotic figures. c High-power view of the area with bone formation at $\times 400$ magnification. High-grade carcinoma cells are admixed with fragments of osseous tissue and multinucleated osteoclasts (arrows).

cells were admixed with immature osseous tissue and multinucleated osteoclasts, supporting the diagnosis of matrix-producing MBC. Breast Biomarkers also showed TNBC.

A computerized tomography scan of the chest showed extensive sclerotic changes in the thoracic spine and upper lumbar spine, anterior right 5 th and 7 th ribs, sacrum and bilateral femoral head and femoral necks, with healing fractures in the 3rd right rib and left 2nd-3rd ribs, with sclerotic areas, as well as a left 6th rib healing fracture. There was a $13 \mathrm{~mm}$ lymph node just lateral to the left axilla, a $28 \mathrm{~mm}$ dense nodule in the right lateral axilla (shown in Fig. 2a) with several more medial sub-centimeter nodes, and several sub-centimeter mesenteric lymph nodes. Right-sided pulmonary bullae had a mass effect shifting the mediastinum slightly leftward. A whole-body bone scan confirmed multiple abnormalities in areas of sclerotic changes. Computerized tomography of the abdomen and pelvis revealed osseous 

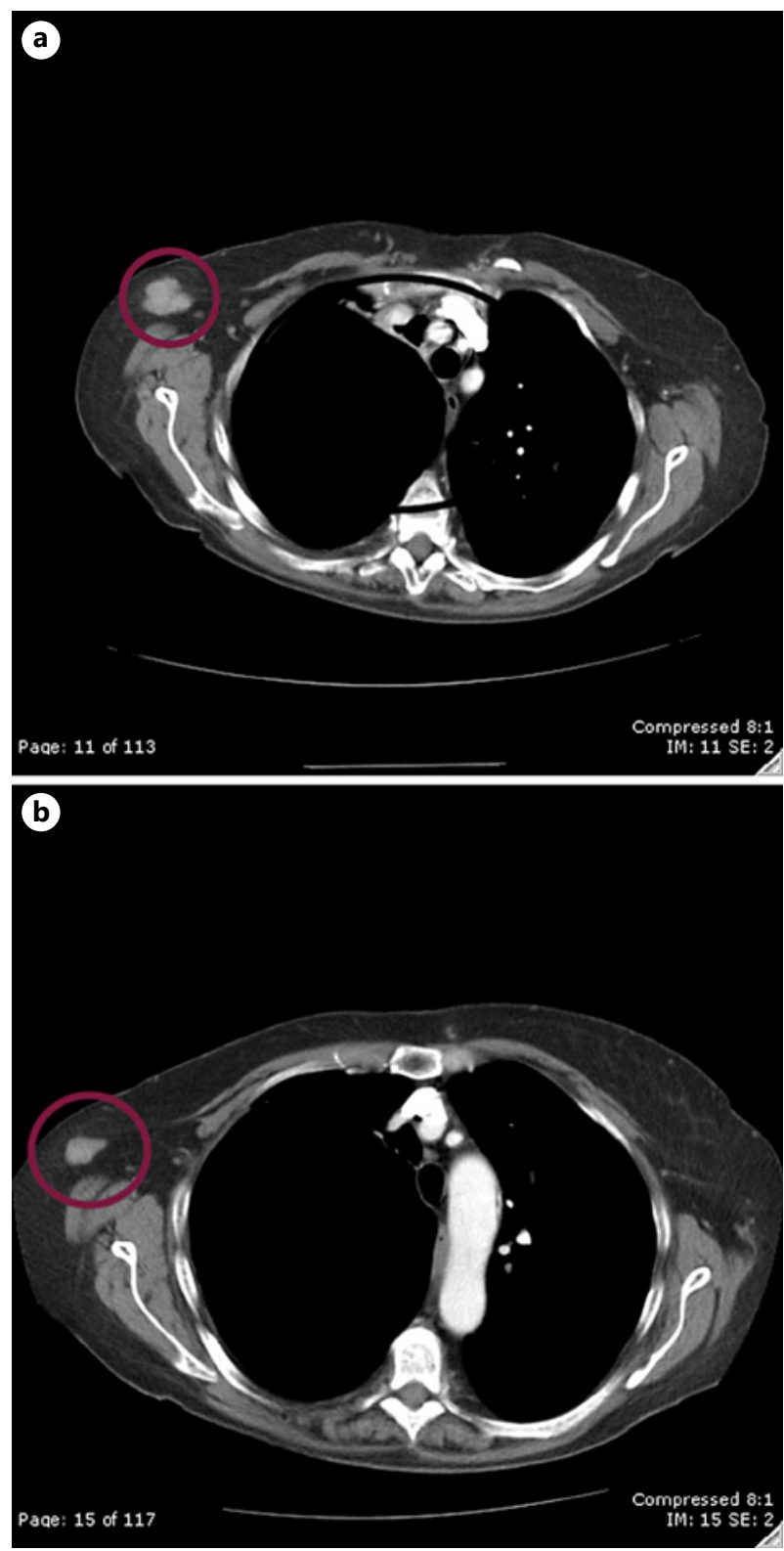

Fig. 2. Radiographic imaging. CT scan of the chest prior to the initiation of pembrolizumab demonstrated a $28 \mathrm{~mm}$ right axillary lymph node (red circle in a). Following 8 cycles of treatment, the lymph node in the right axilla decreased to $16 \mathrm{~mm}$ (red circle in b). CT, computerized tomography.

changes suggestive of metastatic disease but no other specific findings of metastasis. Findings were consistent with a stage IV MBC (pT3NxM1).

Comprehensive genomic profiling using the FoundationOne Heme panel of 405 genes as well as selected introns of 31 genes involved in rearrangements in addition to RNA sequencing of 265 genes was performed. This revealed the presence of the following genomic alterations: AKT1 E17K; KRAS A146T; NUP93 E14K; and TP53 loss, R213. No ERBB2 alterations were noted.

The patient was treatment naïve and her tumor was triple negative, therefore she was eligible for participation on the phase II Study of Pembrolizumab (MK-3475) Monotherapy for Metastatic Triple-NegativeBreastCancer(MK-3475-086/KEYNOTE-086) (NCT02447003). The patient underwent a research biopsy of right axillary lymph node to confirm PD-L1 positivity. The specimen was not decalcified such that it was adequate to perform immunohisto- 
chemistry for PD-L1, which was found to be positive. The patient began treatment with pembrolizumab 200 mg IV every 3 weeks. Restaging scans performed 2 months after initiation of pembrolizumab (status post cycle 2) revealed a decrease in the size of the right axillary lymph node, with otherwise stable disease. After cycle 4, the patient experienced an acute onset of treatment-related immune colitis with bloody diarrhea, which was successfully treated with a tapering course of prednisone, leading to gradual return to the patient's baseline. Following 8 cycles on the clinical trial, restaging scans showed a partial response, with a $16 \mathrm{~mm}$ right lateral axillary lymph node (shown in Fig. 2b). At the 1-year interval, restaging scans documented a persistent yet stable right axillary lymph node. The patient was eligible to remain on study as the lymph node remained stable in size, and alternative therapy options for her subtype of breast cancer were lacking. Due to sustained response to pembrolizumab monotherapy with minimal disease burden restricted to the axilla, the patient underwent a right axillary lymph node dissection with potential curative intent. Final pathology demonstrated cancer in only one out of 19 lymph nodes. The patient proceeded to complete 2 years of pembrolizumab therapy as per protocol. Follow-up CT and bone scans through 32 months of post-therapy showed no evidence of disease recurrence or metastatic disease, and the patient has maintained a good quality of life other than intermittent episodes of colitis that have predated pembrolizumab treatment.

\section{Discussion}

MBC is increasingly recognized as a rare and heterogeneous subtype of breast cancer with an aggressive course and a poor prognosis. The disease typically features higher local recurrence rates and distant metastases, as well as lower disease free and overall survival when compared with more common invasive breast disease, including TNBC [2]. Although MBC may share an origin with ductal carcinoma, the metaplastic component may result from epigenetic changes leading to multiple histologies that adversely impact response to conventional systemic treatment $[3,4]$. Adjuvant radiotherapy should be employed to improve localregional control and overall survival.

More recently, gene profiling has identified MBC as highly heterogeneous tumors at the genomic and transcriptomic levels, and various molecular abnormalities have been recognized that may translate to targetable therapies $[5,6]$. TP53 alterations may be found in up to $75 \%$ of patients [7]. Alterations in the PI3K pathway may be detected in $69 \%$ of patients, which potentially offers an avenue of therapy [8]. Tumoral and immune stromal program death ligand-1 (PD-L1) expression may be identified in a variety of subtypes of breast cancer, but is most common among hormone receptor negative and TNBC [9]. Overexpression of PD-L1, defined as $\geq 5 \%$ cells, may be detected in up to $46 \%$ of patients with MBC, which is significantly higher than other breast tumors [10]. Diffuse PD-L1 staining, defined as $>50 \%$, is noted in approximately $5 \%$ of TNBC [9]. Evidence of clinical activity in pretreated TNBC patients with immune checkpoint inhibition may be related to significant infiltration of TNBC tumors with tumor-infiltrating lymphocytes, and for patients with overexpression of PD-L1, immunotherapy in combination with chemotherapy has demonstrated favorable response rates for patients with MBC [11-14]. Early data has shown antitumor activity of PD1 inhibitors in heavily pretreated estrogen receptor-positive, PD-L1-positive disease, and a phase Ib study found that treatment with pembrolizumab is well-tolerated and may provide durable overall responses in HER2-positive patients with advanced, trastuzumab-resistant, PD-L1-positive breast cancer [15].

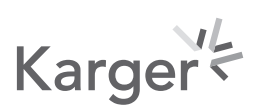




\section{Conclusion}

Prior to immunotherapy trials, patients with metastatic MBC received systemic cytotoxic therapy with minimal benefit in terms of reduction in tumor burden or improvement in disease-related symptoms. In this reported case, the availability of a suitable immunotherapy trial with the PD1 inhibitor pembrolizumab resulted in sustained clinical benefit with preservation of a good quality of life. Further evaluation of the innate features of MBC that confer immunologic vulnerability is needed to better understand which subset of patients derives benefit from immunotherapy.

\section{Acknowledgement}

This research was supported by the Biospecimen Repository Shared Resource(s) of the Rutgers Cancer Institute of New Jersey and Office of Human Research Services (P30CA072720) and a generous gift to the Genetics Diagnostics to Cancer Treatment Program of the Rutgers Cancer Institute of New Jersey and RUCDR Infinite Biologics.

\section{Statement of Ethics}

Subject has given her written informed consent to publish the case (including publication of images). The investigators have permission from the industry-sponsored clinical trial to publish this individual case report. The study protocol was approved by the Institutional Review Board of Rutgers Cancer Institute of New Jersey. The reference number (PMID) is 30475950 , and the clinical trial number is NCT02447003.

\section{Conflict of Interest Statement}

The authors have no conflicts of interest to declare.

\section{Funding Sources}

This research did not receive grants from any funding agency in the public, commercial, or not-for-profit sectors.

\section{Author Contributions}

E.G. - wrote the manuscript; K.M. - interpreted pathology results and provided slides; G.R. - precision medicine initiative pathology review; L.S. - reviewed and provided imaging; L.R.R. - precision medicine initiative case review; F.E. - surgical oncologist for clinical trial enrollment; M.G. - surgical oncologist for management of immunotherapy induced colitis; S.G. - precision medicine initiative genomics analysis; D.T. - medicine oncologist principal investigator of clinical trial enrolled; L.P. - surgical oncologist for metastasectomy; K.T. primary medical oncologist; K.H. - precision medicine initiative genomics analysis; N.C. manuscript development.

\section{Karger'k}




\section{References}

1 Tzanninis IG, Kotteas EA, Ntanasis-Stathopoulos I, Kontogianni P, Fotopoulos G. Management and outcomes in metaplastic breast cancer. Clin Breast Cancer. 2016 Dec;16(6):437-43.

2 Leyrer CM, Berriochoa CA, Agrawal S, Donaldson A, Calhoun BC, Shah C, et al. Predictive factors on outcomes in metaplastic breast cancer. Breast Cancer Res Treat. 2017 Oct;165(3):499-504.

3 Edenfield J, Schammel C, Collins J, Schammel D, Edenfield WJ. Metaplastic breast cancer: molecular typing and identification of potential targeted therapies at a single institution. Clin Breast Cancer. 2017 Feb;17(1):e1-10.

4 Piscuoglio S, Ng CKY, Geyer FC, Burke KA, Cowell CF, Martelotto LG, et al. Genomic and transcriptomic heterogeneity in metaplastic carcinomas of the breast. NPJ Breast Cancer. 2017 Dec 1;3:48.

5 Dave B, Gonzalez DD, Liu ZB, Li X, Wong H, Granados S, et al. Role of RPL39 in metaplastic breast cancer. J Natl Cancer Inst. 2016 Dec 31;109(6):djw292.

6 Abouharb S, Moulder S. Metaplastic breast cancer: clinical overview and molecular aberrations for potential targeted therapy. Curr Oncol Rep. 2015 Mar;17(3):431.

7 Ross JS, Badve S, Wang K, Sheehan CE, Boguniewicz AB, Otto GA, et al. Genomic profiling of advanced-stage, metaplastic breast carcinoma by next-generation sequencing reveals frequent, targetable genomic abnormalities and potential new treatment options. Arch Pathol Lab Med. 2015 May;139(5):642-9.

8 Moulder S, Helgason T, Janku F, Wheler J, Moroney J, Booser D, et al. Inhibition of the phosphoinositide 3-kinase pathway for the treatment of patients with metastatic metaplastic breast cancer. Ann Oncol. 2015 Jul;26(7): 1346-52.

9 Dill EA, Gru AA, Atkins KA, Friedman LA, Moore ME, Bullock TN, et al. PD-L1 expression and intratumoral heterogeneity across breast cancer subtypes and stages. an assessment of 245 primary and 40 metastatic tumors. Am J Surg Pathol. 2017 Mar;41(3):334-42.

10 Joneja U, Vranic S, Swensen J, Feldman R, Chen W, Kimbrough J, et al. Comprehensive profiling of metaplastic breast carcinomas reveals frequent overexpression of programmed death-ligand 1. J Clin Pathol. 2017 Mar; $70(3): 255-9$.

11 Adams S. Dramatic response of metaplastic breast cancer to chemo-immunotherapy. NPJ Breast Cancer. 2017 Mar 29;3:8.

12 Nanda R, Chow LQ, Dees EC, Berger R, Gupta S, Geva R, et al. Pembrolizumab in patients with advanced triplenegative breast cancer: phase Ib KEYNOTE-012 study. J Clin Oncol. 2016 Jul 20;34(21):2460-7.

13 Tray N, Taff J, Adams S. Therapeutic landscape of metaplastic breast cancer. Cancer Treat Rev. 2019 Sep;79: 101888.

14 Al Sayed AD, Elshenawy MA, Tulbah A, Al-Tweigeri T, Ghebeh H. Complete response of chemo-refractory metastatic metaplastic breast cancer to paclitaxel-immunotherapy combination. Am J Case Rep. 2019 Nov 6;20: 1630-5.

15 Rugo HS, Delord JP, Im SA, Ott PA, Piha-Paul SA, Bedard PL, et al. Safety and antitumor activity of pembrolizumab in patients with estrogen receptor-positive/human epidermal growth factor receptor 2-negative advanced breast cancer. Clin Cancer Res. 2018 Jun 15;24(12):2804-11. 